\title{
No variation of physical performance and perceived exertion after adrenal gland stimulation by synthetic ACTH (Synacthen ${ }^{\circledR}$ ) in cyclists
}

\author{
Norbert Baume - Graeme Steel • Tony Edwards • \\ Eric Thorstensen $\cdot$ Benjamin F. Miller
}

Accepted: 9 June 2008 / Published online: 27 June 2008

(C) Springer-Verlag 2008

\begin{abstract}
There is anecdotal evidence that athletes use the banned substance Synacthen ${ }^{\circledR}$ because of its perceived benefit with its associated rise in cortisol. To test the performance-enhancing effects of Synacthen ${ }^{\circledR}$, eight trained cyclists completed two, 2-day exercise sessions separated by 7-10 days. On the first day of each 2-day exercise session, subjects received either Synacthen ${ }^{\circledR}(0.25 \mathrm{mg}$, TX) or placebo (PLA) injection. Performance was assessed by a 20-km time trial (TT) after a 90-min fatigue period on day 1 and without the fatiguing protocol on day 2. Plasma androgens and $\mathrm{ACTH}$ concentrations were measured during the
\end{abstract}

N. Baume

Department of Sport and Exercise Science,

Tamaki Campus, The University of Auckland,

Private Bag 92019, Auckland, New Zealand

G. Steel

Drug Free Sport NZ, 71 Merton Road, St Johns,

Auckland, New Zealand

T. Edwards

Adidas Sports Medicine Centre, Merton Road,

St Johns, Auckland, New Zealand

E. Thorstensen

The Faculty of Medical and Health Sciences,

The Liggins Institute, The University of Auckland,

Private Bag 92019, Auckland, New Zealand

B. F. Miller

Department of Health and Exercise Science, Colorado State University, 201B Moby B Complex,

Fort Collins, CO 80523-1582, USA

N. Baume $(\square)$

Swiss Laboratory for Doping Analyses,

Ch. des Croisettes 22, 1066 Epalinges, Switzerland

e-mail: norbert.baume@chuv.ch exercise bouts as well as the rate of perceived exertion (RPE). Spot urines were analyzed for androgens and glucocorticoids quantification. Basal plasma hormones did not differ significantly between PLA and TX groups before and $24 \mathrm{~h}$ after the IM injection $(P>0.05)$. After TX injection, ACTH peaked at $30 \mathrm{~min}$ and hormone profiles were significantly different compared to the PLA trial $(P<0.001)$. RPE increased significantly in both groups as the exercise sessions progressed $(P<0.001)$ but was not influenced by treatment. The time to completion of the TT was not affected on both days by Synacthen ${ }^{\circledR}$ treatment. In the present study, a single IM injection of synthetic ACTH did not improve either acute or subsequent cycling performance and did not influence perceived exertion. The investigated urinary hormones did not vary after treatment, reinforcing the difficulty for ACTH abuse detection.

Keywords ACTH · Cortisol · Androgens · Doping · Cycling

\section{Introduction}

Synacthen ${ }^{\circledR}$ (tetracosactide), a long-acting synthetic $\beta^{1-24}$ corticotropin, exhibits the same activity as natural adrenocorticotropic hormone (ACTH) (Johnson and Renn 2006; Sewer and Waterman 2003). A depot Synacthen test is used as a clinical measure of adrenal function in those with hypothalamic-pituitary-adrenal (HPA) axis disorders and allows the evaluation of diseases such as Addison's disease (Nieman and Chanco Turner 2006; Wood et al. 1965). As evident from recent high-profile drug seizures, it is thought that Synacthen ${ }^{\circledR}$ is used by some competitive athletes to acutely stimulate secretion of glucocorticoids like cortisol (hydrocortisone) and cortisone by the adrenal cortex. These 
two hormones are naturally occurring steroid hormones, which exist in equilibrium with cortisol, being the biologically active form (Ricketts et al. 1998). As glucocorticoid receptors are ubiquitous in the body (Rook 1999), cortisol has a large effect on diverse physiologic systems. Glucocorticoids are primarily used for their anti-inflammatory (Rook 1999) and analgesic properties in order to decrease pain and increase feelings of well-being (Bogdanov and Yarushkina 2004). Glucocorticoids are also becoming more popular for the treatment of acute and chronic asthmatic symptoms (Fitch 1986). Due to the higher physical exertion, pain and incidence of injuries in some sports, glucocorticoids have also been widely used for improving performance since the 1960s (Dvorak et al. 2006; Nichols 2005). Administration of these substances (except topically-as defined) is prohibited by the World Anti-Doping Agency (WADA), while their use is allowed for local treatment and legitimate medical purposes (WADA 2008).

Uncertainty regarding the performance-enhancing effect of acute adrenocorticotropin administration stems from the relatively short duration of their effects on increased steroid hormones levels and questions about whether cortisol peak is performance enhancing considering short-term and intense exercise. Some authors hypothesize that the positive lipolytic effect of ACTH could increase endurance performance by increasing free fatty acid availability (Reed et al. 1987), but this is doubtful given the results of other studies using high-fat diets to increase FFA availability (Burke and Hawley 2002).

One study directly evaluated the efficacy of adrenocorticotropins on exercise performance (Soetens et al. 1995). Professional cyclists performed $1 \mathrm{~h}$ of steady-state exercise followed by an incremental test to exhaustion in a doubleblind design with 1-mg ACTH or placebo. The authors found no increase in maximal performance the day of or the day following exercise, although during the submaximal bout there was a decreased feeling of fatigue. Clearly, more studies are necessitated to evaluate whether or not synthetic ACTH is performance enhancing. This information is one of the three criteria defined by the WADA to justify the presence or the absence of a substance on the banned list (WADA 2008). As such, the statement that a substance has been determined not to be performance enhancing could possibly discourage its further abuse because of its perceived non-benefit.

In addition to the performance effects, detection of Synacthen ${ }^{\circledR}$ administration remains a challenge for the antidoping laboratories. Very recently, a direct method has been published allowing the mass spectrometric Synacthen ${ }^{\circledR}$ detection in human plasma (Thevis et al. 2006). However, urine is still the preferred biological fluid used for antidoping test and the finding of any urinary secondary markers indicative of Synacthen ${ }^{\circledR}$ abuse by athletes would be very useful. Regarding glucocorticoid administration, urinary ratios between cortisol and its precursors and/or metabolites have been proposed without conclusive results (Phillipov 1998; Vogeser et al. 2001). Even if it is known that use of ACTH could affect the urinary endogenous profile of anabolic compounds like testosterone and its related compounds (androgens) (Tamm et al. 1966; Wilson and Lipsett 1966), there is currently no published data examining the value of blood and/or urine profiles for detection of synthetic adrenal manipulation.

The aim of this study is to evaluate whether synthetic ACTH affects both acute and subsequent exercise performance, since there could be effects beyond the time course of administration, and perceived exertion. In addition, urinary and plasma endogenous steroids and glucocorticoids were evaluated to examine any potential secondary marker of synthetic ACTH administration.

\section{Materials and methods}

\section{Subjects}

The study was conducted in the Department of Sport and Exercise Science at the University of Auckland, after the approval of the protocol by the Ministry of Health's Northern Y Ethics Committee (New Zealand). Eight healthy male volunteers, aged between 20 and 35 and trained for cycling (average training distance of $200 \mathrm{~km}$ per week) were recruited and provided informed written consent for participation in the study. Volunteers filled in a medical history questionnaire to exclude those with hormonal dysfunction, cardiovascular disease or history of having used any controlled substances or medication known to affect steroid metabolism or to interact with Synacthen ${ }^{\circledR}$. Because of the side effects, subjects with asthma or any allergy history were excluded. Before enrollment, investigators ensured, in collaboration with Drug Free Sport New Zealand (DFSNZ), that no cyclists were currently competing or part of a registered testing pool.

\section{Screening test}

Upon inclusion in the study, a 9-site skin fold measurement was used to define the participants body composition (Marfell-Jones 2000), and maximal aerobic capacity was determined by performing a standard $V \mathrm{O}_{2 \max }$ test, (initial workload of $150 \mathrm{~W}$ with incremental steps of $30 \mathrm{~W}$ every minute) until volitional fatigue (peak power output, PPO). Expired gases were analyzed continuously (AEI Moxus II gas-analysis system, Naperville, IL) and averaged for each 30 -s period. Heart rate (HR) was monitored every $30 \mathrm{~s}$ (Polar Electro, Kempele, Finland) and the RPE scale, used 
as a measure of perceived exertion during exercise (Borg 1982), was administered at the end of each stage. Criteria used for a maximal exercise test included a plateau of $\mathrm{VO}_{2}$, RER $\geq 1.05$, RPE $>18$, and a HR within 10 beats of the participant's age-predicted maximum. Participants also completed a familiarization $20-\mathrm{km}$ TT before the exercise testing days started.

\section{Exercise testing}

The trial took place during the high cycling season in New Zealand (November-February) meaning that the subjects were well trained. In a randomized, double-blind, crossover design, participants completed two, 2-consecutive day, exercise sessions, each separated by $7-10$ days (week 1 and week 2). On the first day of the testing sessions (day 1), participants received either one $0.25-\mathrm{mg}$ TX or an isovolumetric PLA IM injection. Only the physician administering the drug had the key for injection order. The physician brought to the laboratory a prepared syringe and left the laboratory after the injection. No injection was given on the second and following day of testing (day 2). The same pre-analytical conditions were applied on both days.

The participants reported to the laboratory at 7:00 a.m. having completed light (less than $1 \mathrm{~h}$ at low intensity) or no training the day before. Upon arrival, the participant's body weight was recorded before lying down in a semi-supine position for a 1-h rest period. A catheter was placed in a superficial arm vein for a baseline blood sample $\left(t_{0}\right)$ with subsequent samples at $30 \mathrm{~min}\left(t_{1}\right)$ and $1 \mathrm{~h}\left(t_{2}\right)$. After $30 \mathrm{~min}$ of rest, the IM injection (TX or PLA) was administered in the gluteal region. Thirty minutes later, the participants transferred to the cycle ergometer (Kingcycle, Sands, UK) for calibration followed by the exercise protocol (Fig. 1).

On day 1, a fatiguing protocol and 20-km all out TT was performed to mimic competition conditions. The initial 90min fatiguing period was performed at $50 \%$ PPO, interspersed with three 1-min sprints at $90 \%$ PPO at 10, 35 and $80 \mathrm{~min}$, and two 4-min sprints at $70 \%$ PPO at 45 and $70 \mathrm{~min}$. Immediately at the end of the 90-min fatigue period, participants performed a maximal effort $20 \mathrm{~km}$ TT with only distance completed information exposed to the participant and time to completion recorded by the investigator. HR was monitored continuously (Polar Precision Performance Software, Polar Electro Oy, Finland) while the RPE scale was administered every 10 min during the fatigue period and every $5 \mathrm{~min}$ (although subjects were not told the time frame) during the $20-\mathrm{km}$ TT by pointing to the Borg scale.

The next day (day 2) the subjects reported to the laboratory at 9:00 a.m. and repeated the 20-km TT with the same pre-exercise conditions described above, but without the IM injection. A 7-min warm-up at $40 \%$ of PPO was com-

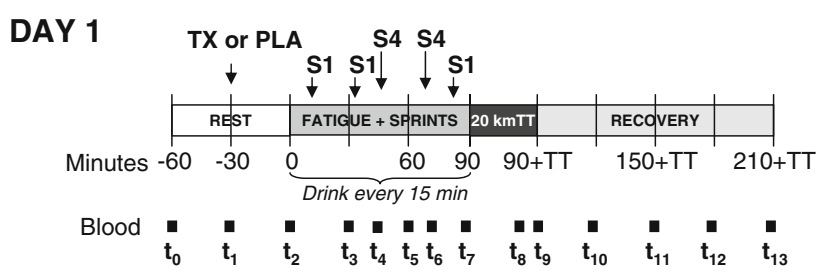

DAY 2

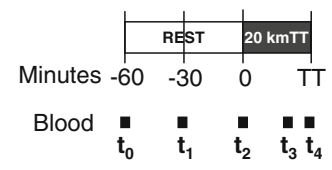

Fig. 1 Schematic representation of the 2-day protocol. On day 1, the IM injection of Synacthen ${ }^{\circledR}(T X)$ or placebo (PLA) happened $30 \mathrm{~min}$ prior to the beginning of the fatigue session which was interspersed with three 1-min sprints $(S 1)$ at $90 \%$ PPO and two 4-min sprints $(S 4)$ at $70 \%$ PPO. The 1-h resting period took place on both days while the 2 -h recovery period was done only on day 1 after the $20-\mathrm{km}$ time trial $(20-\mathrm{km} \mathrm{TT})$. Fourteen and five blood samples were taken on day $1\left(t_{0}-\right.$ $\left.t_{13}\right)$ and day $2\left(t_{0}-t_{4}\right)$, respectively

pleted before performing a maximal effort $20-\mathrm{km}$ TT. At the end of experiment, subjects were asked in which trial they thought they had received the Synacthen ${ }^{\circledR}$ injection based on their own feelings.

\section{Diet control}

Dietary controls were put in place to mimic real competition habits. A diet log was completed for the $24 \mathrm{~h}$ leading up to the first test and participants were requested to reproduce the first record as closely as possible for the day 2 and for both days in the second session. At 10:00 p.m., the evening before the test, participants were instructed to consume a shake $(1,285 \mathrm{KJ}, 56 \mathrm{~g}$ CHO, $5 \mathrm{~g}$ Fat, and $12 \mathrm{~g}$ Pro, Pro4 Nutrition, Auckland, NZ). Finally, participants were given an energy bar (1,109 KJ, $45.6 \mathrm{~g} \mathrm{CHO}, 4.8 \mathrm{~g}$ Fat, and $8.5 \mathrm{~g}$ Pro, Pro4 Nutrition, Auckland, NZ) to consume at 7:00 the morning of the experiment. Subjects drank $500 \mathrm{ml}$ of water during the 1-h resting period and a measured aliquot $(15 \mathrm{~g}$ ) of carbohydrate drink dissolved in $250 \mathrm{ml}$ water was provided to subjects every $15 \mathrm{~min}$ during the 90 -min fatigue exercise period. On day 1 , participants consumed a post-exercise shake $(1,285 \mathrm{KJ}, 56 \mathrm{~g}$ CHO, $5 \mathrm{~g}$ Fat, and $12 \mathrm{~g}$ Pro, Pro4 Nutrition, Auckland, NZ) 1-h after the completion of the exercise protocol.

\section{Stimulation test}

Volunteers were randomly injected with either $0.25 \mathrm{mg}$ of tetracosactide ( $\beta^{1-24}$-corticotropin) dissolved in $1 \mathrm{ml}$ of excipient (Synacthen ${ }^{\circledR}$, Novartis, Basel, Switzerland) or an isovolumetric dose of a sterile saline solution (sodium chloride injection BP $0.9 \%$, Baxter, Australia). Injections were 
done intramuscularly in the gluteal region. The $0.25-\mathrm{mg}$ dose of Synacthen ${ }^{\circledR}$ injected in this experiment is the usual dose the endocrinologists used for undertaking adrenal function testing and is thought to be almost completely devoid of side effects. Nevertheless, side effects including vomiting, nausea, dizziness, flushing, pain or swelling and discomfort at injection site, dyspnea, pruritis, urticaria, bronchospasm, angioedema, or malaise are rarely observed (Medsafe 2006). These reactions occur mostly in the 30min after the injection and that is the reason why the participants stayed supine for $30 \mathrm{~min}$ after the IM injections and under the doctor's supervision.

\section{Blood analyses}

During the 90-min exercise bout, blood was sampled at various intervals $\left(t_{2}=0 \mathrm{~min}, t_{3}=30 \mathrm{~min}, t_{4}=45 \mathrm{~min}\right.$, $\left.t_{5}=60 \mathrm{~min}, t_{6}=74 \mathrm{~min}, t_{7}=90 \mathrm{~min}\right)$ and in the course of the $20-\mathrm{km}$ TT, blood was sampled at $10 \mathrm{~km}\left(t_{8}\right)$, and at $19 \mathrm{~km}\left(t_{9}\right)$. On day 1,4 blood samples were withdrawn every $30 \mathrm{~min}$ during the 2 -h recovery period $\left(t_{10}-t_{13}\right)$. Blood was collected in two chilled vacutainer tubes (BD Diagnostics, Franklin Lakes, NJ, USA) that were immediately spun at 3,000 rpm for $8 \mathrm{~min}$ at RT and instantly stored at $-80^{\circ} \mathrm{C}$ until further analysis.

Plasma cortisol was measured in $20 \mu \mathrm{l}$ of plasma using the Elecsys ${ }^{\circledR} 1010$ Cortisol kit from Roche (Roche Diagnostics, Rotkreuz, Switzerland). Two internal controls and three external quality controls (Lyphochek Immunoassay Plus Control, trilevel) were measured with each batch. The assay coefficients of variation are $2.6 \%$ (intra-assay) and $5.6 \%$ (interassay), and the sensitivity is $0.5 \mathrm{nmol}^{-1}$.

ACTH concentrations were measured by radioimmunoassay (ACTH 125I RIA kit, Diasorin S.p.A., Saluggia, Italy). Two internal and three external controls were used for each batch tested. The assay coefficients of variation are $12.5 \%$ (intra-assay) and 6.0\% (interassay), and the sensitivity is $3 \mathrm{pmol}^{-1}$.

Plasma steroids were measured using liquid chromatography/mass spectrometry (LC/MS/MS). The internal standards were: cortisol-d2 for cortisol; corticosterone-d8 for androstenedione (Adione), dehydroepiandrosterone (DHEA), and testosterone (T). $100 \mu$ l of internal standard (20 $\mathrm{ng} \mathrm{ml}^{-1}$ in water) was added to $200 \mu \mathrm{l}$ plasma. Steroids were extracted using $1 \mathrm{ml}$ of ethyl acetate. After removal of the organic supernatant, samples were dried (Heto freeze drier, Heto Lab Equipment, Denmark), resuspended in $100 \mu \mathrm{l}$ of mobile phase (80\% methanol and $20 \%$ water), and transferred to HPLC injector vials. $25 \mu \mathrm{l}$ was injected onto an HPLC mass spectrometer system consisting of a Surveyor MS pump and autosampler followed by an Ion Max APCI source on a Finnigan TSQ Quantum Ultra AM triple quadrapole mass spectrometer all con- trolled by Finnigan Xcaliber software (Thermo Electron Corporation, San Jose, CA, USA). The mobile phase was isocratic, flowing at $600 \mu \mathrm{min}^{-1}$ through a Luna $3 \mu \mathrm{C} 18(2) 100 \mathrm{~A} 250 \times 4.6$ column at $40^{\circ} \mathrm{C}$ (Phenomenex, Auckland, New Zealand). Retention times were: cortisol $6.0 \mathrm{~min}$; Adione $8.1 \mathrm{~min}$; testosterone $8.7 \mathrm{~min}$, DHEA $9.3 \mathrm{~min}$. Ionization was in positive mode and Q2 had 1.2 mTorr of argon for all steroids. The mass transitions followed, for internal standard and steroid, respectively, were: cortisol-d2 $365.3 \rightarrow 121.2$ at $28 \mathrm{~V}$ and cortisol $363.3 \rightarrow 122.2$ at $28 \mathrm{~V}$; corticosterone-d8 $355.3 \rightarrow 125.2$ at $24 \mathrm{~V}$, DHEA $271.2 \rightarrow 213.1,197.0$ at $18 \mathrm{~V}$, testosterone $289.2 \rightarrow 97.1 .0$ at $27 \mathrm{~V}$, and androstenedione $287.1 \rightarrow 97.1,109.1$ at $28 \mathrm{~V}$. External quality controls were run at the beginning, in the middle, and at the end of each LC/MS/MS assay. Mean values were $31.1 \mathrm{ng} \mathrm{ml}^{-1}$ (cortisol), $1.4 \mathrm{ng} \mathrm{ml}^{-1}$ (DHEA), $1.1 \mathrm{ng} \mathrm{ml}^{-1}$ (Adione) and $355.4 \mathrm{pg} \mathrm{ml}^{-1}$ (free T). The assay coefficients of variation were between 10 and $25 \%$ (intra-assay) and between 8.0 and 20\% (interassay). Blank urines were also run during the assays as negative controls.

Quantification of urinary steroid and glucocorticoids

Quantitative analyses of urinary steroids and glucocorticoids glucuronides were performed using similar procedures of a previously reported method (Mazzarino et al. 2006). Briefly, $1 \mathrm{ml}$ of urine was added with $20 \mu \mathrm{l}$ of internal standard (IS) mix (etiocholanolone-d5 (etio-d5, 40 $\mu \mathrm{g} \mathrm{ml}^{-1}$ ), testosterone-d3 (testo- $\mathrm{d} 3,4.5 \mu \mathrm{g} \mathrm{ml}^{-1}$ ), cortisol$\mathrm{d} 4$ (HC-d4, $10 \mu \mathrm{g} \mathrm{ml}^{-1}$ ) and androsterone-d4 glucuronide (andro-d4, $40 \mu \mathrm{g} \mathrm{ml}^{-1}$ ). Sample was then extracted on a C18 column and methanolic eluate evaporated. After hydrolysis, $2 \mu \mathrm{l}$ of the derivatized extract was injected into a GC-MS 5890/5973 (Agilent Technologies Inc., CA, USA). The analyses were performed in single ion monitoring (SIM) mode with the following ions ( $\mathrm{m} / \mathrm{z}$ ): 438 (androd4); 439 (etio-d5); 435 (testo-d3); 434 [androsterone, etiocholanolone and dihydrotestosterone (DHT)]; 432 [T, epitestosterone (EpiT) and DHEA]; 430 (Adione); 241 (5 $\alpha$ androstan- $3 \alpha-17 \beta$-diol and $5 \beta$-androstan- $3 \alpha-17 \beta$-diol); 522 [11-OH etiocholanolone; 343 (11-keto etiocholanolone)]; 636 (HC-d4 and tetrahydrocortisol); 632 (cortisol); 615 (cortisone), 562 (tetrahydrocortisone) and 548 (11deoxycortisol). A five-point calibration curve was established using available reference material with the urinary concentration range of $5-500 \mathrm{ng} \mathrm{ml}^{-1}$ for T, EpiT, DHT, DHEA, $\alpha$-diol, $\beta$-diol, Adione, 11-keto-etio, 11-OH etio, cortisone, cortisol, tetrahydrocortisone $\left(5-500 \mathrm{ng} \mathrm{ml}^{-1}\right.$, $\mathrm{R}^{2}>0.965$ ). For androsterone, etiocholanolone and tetrahydrocortisol, the urinary concentration range of 100 $2,000 \mathrm{ng} \mathrm{ml}^{-1}$ was used for calibration curve (1002,000 $\mathrm{ng} \mathrm{ml}^{-1}, \mathrm{R}^{2}>0.998$ ). 
Table 1 Mean anthropometrics and performance characteristics measured during the screening test $(n=8)$

\begin{tabular}{ll}
\hline & Mean \pm SD \\
\hline Age (years) & $28.5 \pm 4.3$ \\
Weight $(\mathrm{kg})$ & $79.0 \pm 7.4$ \\
Height $(\mathrm{m})$ & $1.81 \pm 0.07$ \\
BMI & $24.1 \pm 1.8$ \\
Fat mass $(\%)$ & $13.7 \pm 3.9$ \\
$V \mathrm{O}_{2}$ max $\left(\mathrm{ml} \mathrm{kg}^{-1} \mathrm{~min}^{-1}\right)$ & $63.7 \pm 4.5$ \\
Peak power output $(\mathrm{W})$ & $428 \pm 54$ \\
\hline
\end{tabular}

\section{Statistical analysis}

All statistical analyses were performed on Matlab ${ }^{\circledR}$ version 7.3.0, with Statistics Toolbox version 5.3. Deviations from normality were evaluated with a Jarque-Bera two-sided goodness-of-fit test on data collected before treatment. When necessary, androgens data were log-transformed to be closer to a normal distribution. A four-way analysis of variance (ANOVA) with repeated measures was then used to test the effect of the factors treatment: [TX, PLA], subject: $[1, \ldots, 8]$, session: $[1,2]$ and time: $[-60, \ldots, 243]$ on the mean RPE values and plasma ACTH, cortisol, DHEA, Adione and $\mathrm{T}$ concentrations. A two-sample $T$-test was used to compare mean basal plasma values of plasma cortisol, ACTH, DHEA, Adione and free T before the IM injection. A two-sample Kolmogorov-Smirnov test was used to compare the time to completion between the placebo and TX groups, since this parameter was not normally distributed. Significance was set at $P \leq 0.05$.

\section{Results}

The mean anthropometrics values and performance characteristics measured on the eight subjects are shown in Table 1.

To mimic the stochastic nature of a cycling race, five sprint sessions were introduced in the fatigue period before the TT. The continuous HR monitoring illustrates clearly the different phases in the fatiguing exercise prior to the 20$\mathrm{km}$ TT (Fig. 2). The superposition of the HR profiles from each condition (PLA and TX) is an indication that the participants were exercising at the same relative exercise intensity. The abrupt HR drop-off corresponded to short rest room interruptions during the efforts without standardized timing.

On day 1 , the total number of blood samples was 217,31 before treatment, 186 after treatment ( 7 withdrawals failed for technical reasons). No evidence against normality was found on the plasma cortisol and ACTH data obtained

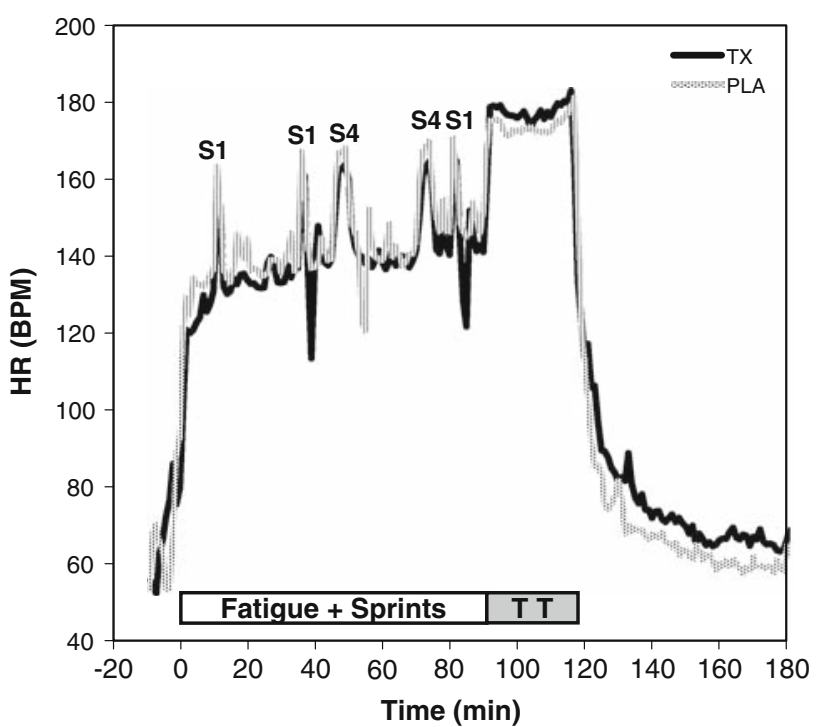

Fig. 2 Continuous heart rate $(H R)$ recordings of the same subject during day 1 sessions under the Synacthen ${ }^{\circledR}(T X$, dark line) and placebo (PLA, gray line) conditions. The fatigue and sprints as well as the 20$\mathrm{km}$ TT phases are indicated. S1 1-min sprints; $S 4$ 4-min sprints

before treatment $(P=0.86, P=0.89$, respectively; $n=31)$. Plasma cortisol profiles measured on day 1 during the PLA and TX trials were similar $(P=0.40)$ before the IM injection $\left(t_{0}\right.$ and $\left.t_{1}\right)$ with a mean $t_{0}$ value of $478.6 \mathrm{nmol}^{-1}$ and $517.0 \mathrm{nmol}^{-1}$ in the PLA and TX trial, respectively (Fig. 3a). All the factors considered in the ANOVA of cortisol showed a significant $P$ value $(<0.001)$ and the percentage of explained cortisol variance by treatment (PLA or TX), subject, week (1 or 2) and sampling time were 45, 5, 2 and $14 \%$, respectively. Mean basal ACTH concentration before the IM injection $\left(t_{0}\right)$ was similar in PLA and TX groups (6.6 and $6.5 \mathrm{pmol}^{-1}$, respectively), and ANOVA revealed that ACTH concentration was significantly influenced by sampling time $(P<0.001)$ and treatment $(P=0.002)$ (Fig. 3c). Twenty-four hours after the IM injection, the plasma cortisol (PLA, $361.3 \mathrm{nmol}^{-1}$ vs. TX, $353.4 \mathrm{nmol}^{-1}$ ) and ACTH (PLA, $5.4 \mathrm{pmol}^{-1}$ vs. TX, $4.7 \mathrm{pmol}^{-1}$ ) returned to resting concentrations and no statistical difference was found between the PLA and TX conditions (Fig. 3b, d).

Plasma DHEA, Adione and free $\mathrm{T}$ were quantified and profiles of day 1 are shown in Fig. $4 a$, b, c, respectively. Evidence against normality was found for DHEA and Adione, but not for free $\mathrm{T}$, for the data collected at time $t_{0}=-60$ and $t_{1}=-30 \mathrm{~min} \quad(P=0.0040, \quad P=0.035$, $P=0.70$, respectively; $n=31$ ), whereas no evidence against normality was found for DHEA and Adione after log-transformation $(P=0.33$ and $P=0.15$, respectively). All DHEA and Adione data were thus further studied on a $\log$-scale. As cortisol, $t_{0}$ and $t_{1}$ were not significantly different for the three hormones with PLA and TX mean basal 
Fig. 3 Mean TX (solid line, filled triangle) and PLA (dashed line, open square) plasma cortisol and ACTH values ( \pm SEM; $n=8$ subjects) measured during day 1 (a and $\mathbf{c}$ ) and day 2 (b and d). $\mathrm{F}+\mathrm{S}=$ fatigue and sprints period; $T T=20-\mathrm{km}$ time trial period
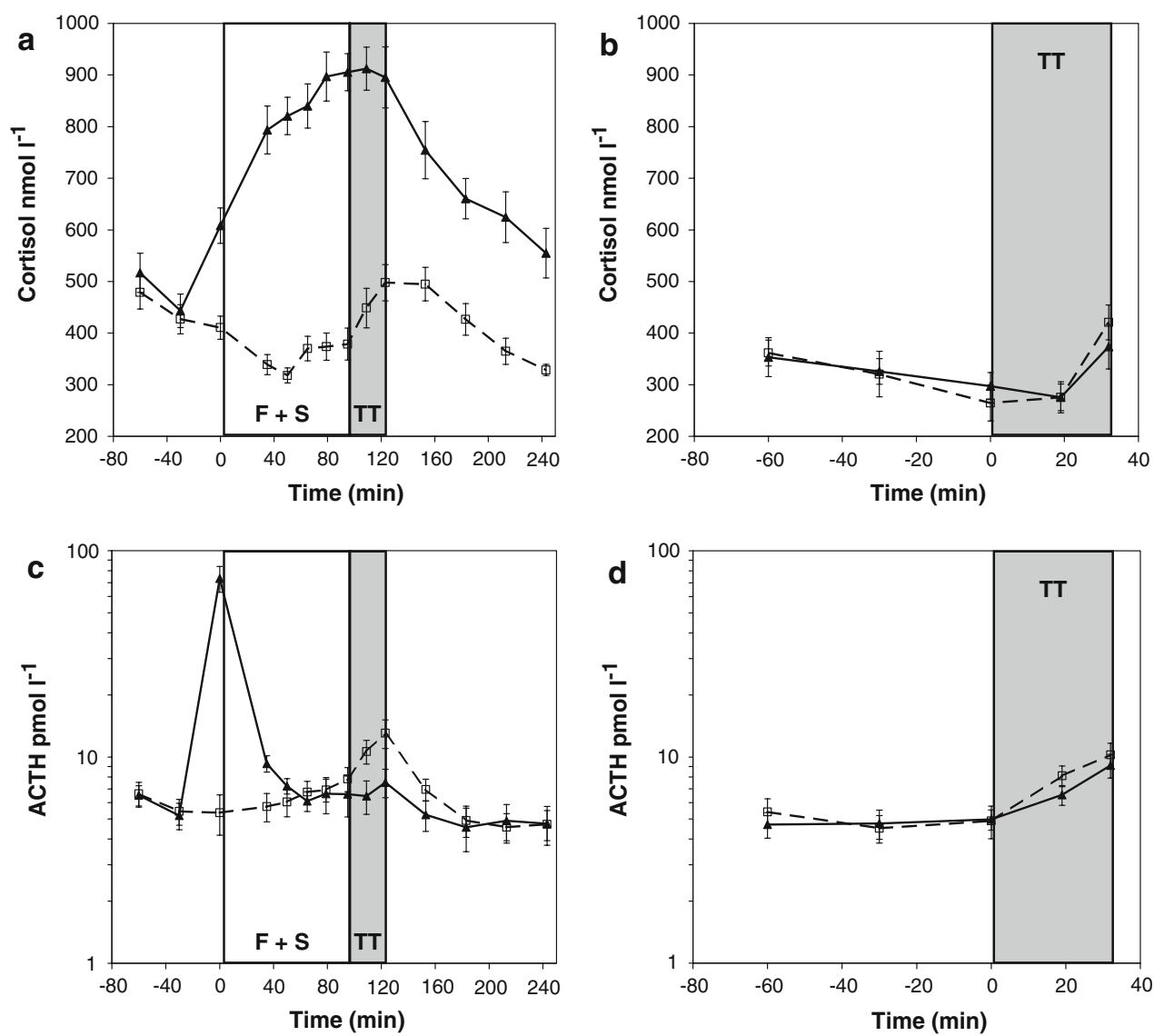

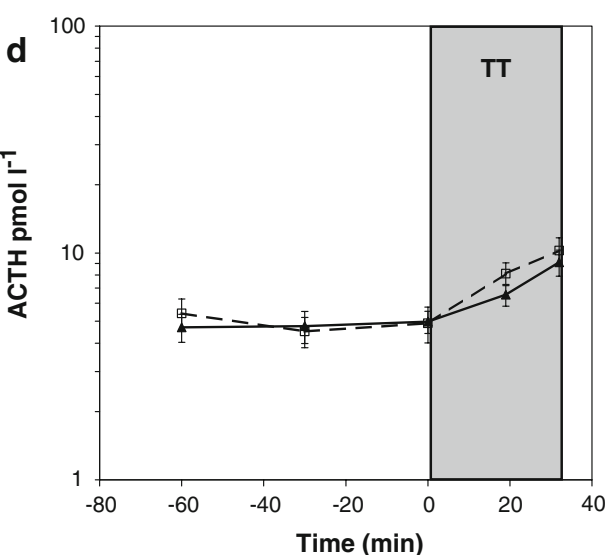

values of 4.9 and $5.4 \mathrm{ng} \mathrm{ml}^{-1}$ (geometric mean $\mathrm{GM}=4.6$ and $\mathrm{GM}=4.9 \mathrm{ng} \mathrm{ml}^{-1} ; P=0.67$ ) for DHEA, 0.69 and $0.78 \mathrm{ng} \mathrm{ml}^{-1} \quad\left(\mathrm{GM}=0.63\right.$ and $\mathrm{GM}=0.70 \mathrm{ng} \mathrm{ml}^{-1}$; $P=0.51)$ for Adione and 2.4 and $2.5 \mathrm{ng} \mathrm{ml}^{-1}(P=0.69)$ for free T. $24 \mathrm{~h}$ after the IM injection, the three hormones returned to initial concentrations with no statistical difference between PLA and TX trials (data not shown). ANOVA of DHEA, Adione and free plasma T revealed a significant effect of treatment (PLA or TX), sampling time and subject.

Performance was measured from time to completion of the $20-\mathrm{km}$ TT. On day 1 , the mean time $( \pm \mathrm{SD})$ to completion was $28.19 \pm 1.67 \mathrm{~min}$ and $28.29 \pm 1.88 \mathrm{~min}$ in PLA and TX trials, respectively (Fig. 5), without any statistical difference between the trials $(P=0.93)$. On day 2 , the mean time $( \pm \mathrm{SD})$ to completion was $27.28 \pm 1.47 \mathrm{~min}$ in the PLA trial and $27.01 \pm 1.40 \mathrm{~min}$ in the TX trial with no statistical difference $(P=0.99)$ between the trials. Participants completed the $20-\mathrm{km}$ TT significantly faster on day 2 than on day 1 of the protocol in both conditions (PLA and TX) $(P=0.023)$. Indeed, all subjects decreased their time to completion between 0.8 and $9.3 \%$ in the TX trial and between 1.2 and $7.6 \%$ in PLA the condition.

RPE increased over time independently of TX or PLA injection $(P<0.001)$ for both day 1 (Fig. 6a) and day 2
(Fig. 6b). RPE was significantly influenced by treatment $(P=0.018$, TX significantly higher than PLA) and by week ( $P=0.011$, significantly lower RPE in the second session). No evidence of interaction of treatment and time was found $(P=0.99)$ in PLA compared with TX trial. At the end of the experiment and according to their subjective feelings, 4 participants (50\%) guessed correctly in which session they received the Synacthen ${ }^{\circledR}$ injection.

Spot urines collected from the eight subjects during the testing days were analyzed for androgens and glucocorticoids quantification. Only individual T/epiT ratio values (Fig. 7a, b) for PLA and TX session are shown. Regarding T/epiT ratio, no abnormal intra-individual variation was found according with the model developed by Sottas et al. (2008). Intra-individual T/epiT ratios had a coefficient of variation fluctuating between 6.6 and $16.1 \%$ in the PLA conditions and between 5.9 and $22.1 \%$ in TX trial.

\section{Discussion}

The purpose of this study was first to evaluate the effect of a single IM injection of synthetic ACTH $(0.25 \mathrm{mg}$ Synacthen ${ }^{\circledR}$ ) on cycling maximal performance assessed by the completion of a $20-\mathrm{km}$ TT and on RPE, and second to 
Fig. 4 Mean TX (solid line, filled triangle) and PLA (dashed line, open square) plasma DHEA (a), Adione (b) and free $\mathrm{T}$ (c) values ( \pm SEM; $n=8$ subjects) measured during day 1 of the sessions
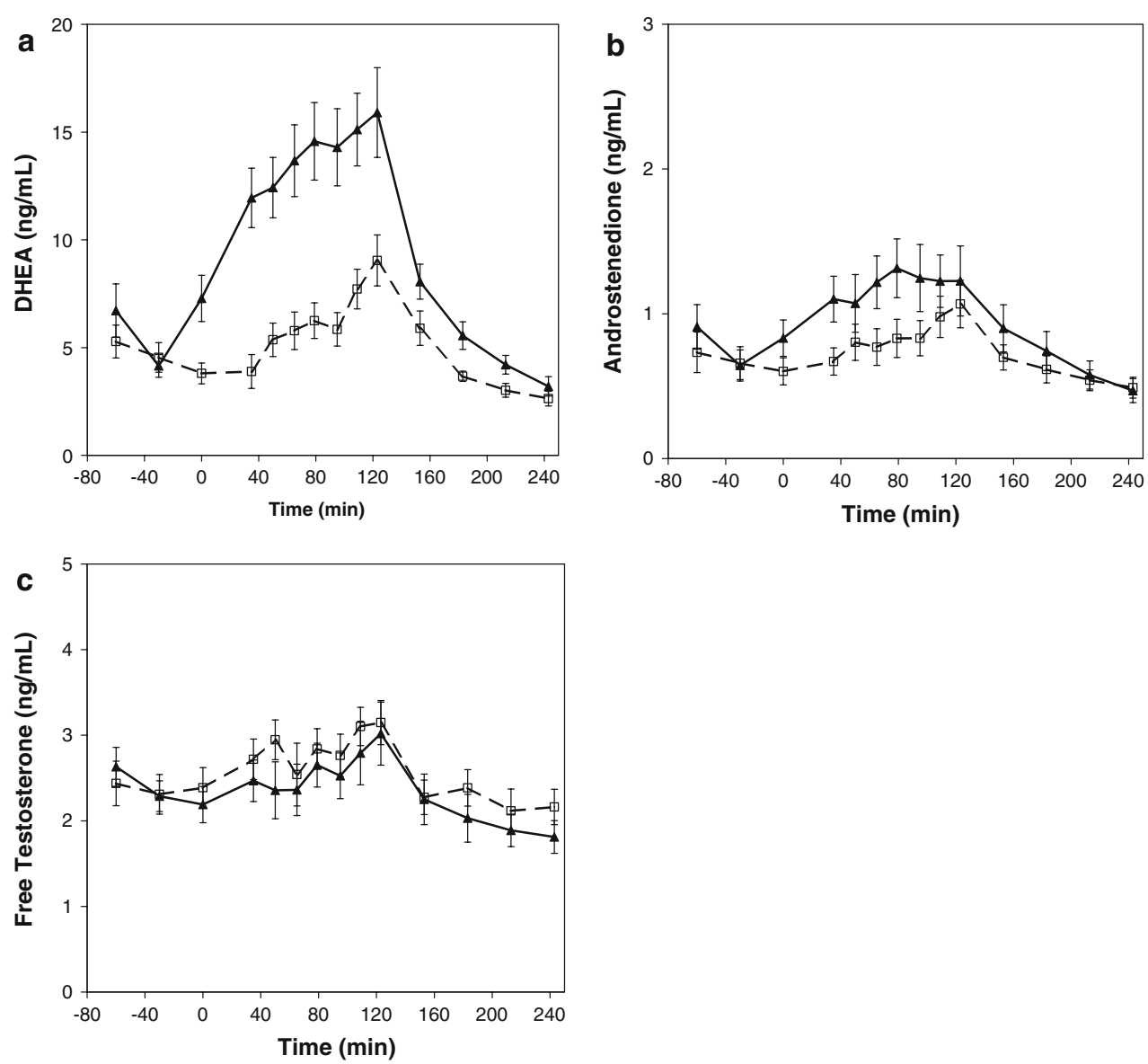

identify any blood and/or urinary secondary markers of synthetic ACTH abuse. The results of the present study do not support the acute use of Synacthen ${ }^{\circledR}$ as a performanceenhancing agent and are in agreement with the only other study that has been made in this field. Moreover, none of the investigated biomarkers in both biological fluids allowed a differentiation between the treated and the placebo session.

General considerations about Synacthen ${ }^{\circledR}$

In the last few decades, glucocorticoids have become very popular among endurance athletes for their anti-inflammatory and analgesic effects, and their ability to improve athlete alertness (Abdi et al. 2007; Polettini et al. 1998). Thus, athletes are able to train and compete while they are injured and/or tired. For these reasons, all glucocorticoids administered orally, rectally, intravenously or intramuscularly are prohibited by the WADA (WADA 2008). Recent research and development of technologies have allowed scientists to detect exogenous glucocorticoids and also to differentiate between endogenous and exogenous corticoids (Bourgogne

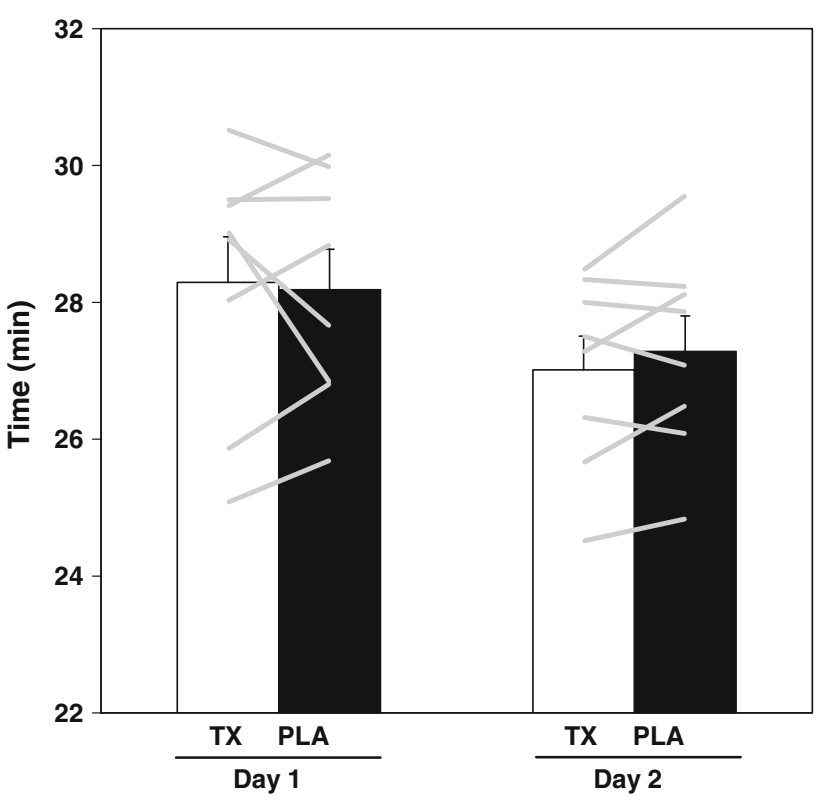

Fig. 5 Time to completion of the 20-km TT on day 1 and day 2 after Synacthen $^{\circledR}(T X)$ or saline (PLA) IM injection. Values are means \pm SEM for 8 subjects. Individual data are also plotted for all subjects on both days 
Fig. 6 Mean RPE values ( \pm SEM; $n=8$ subjects) recorded during day 1 (a) and day 2 the dashed line (open square) and TX values by the solid line (filled triangle) (b). PLA data are indicated by
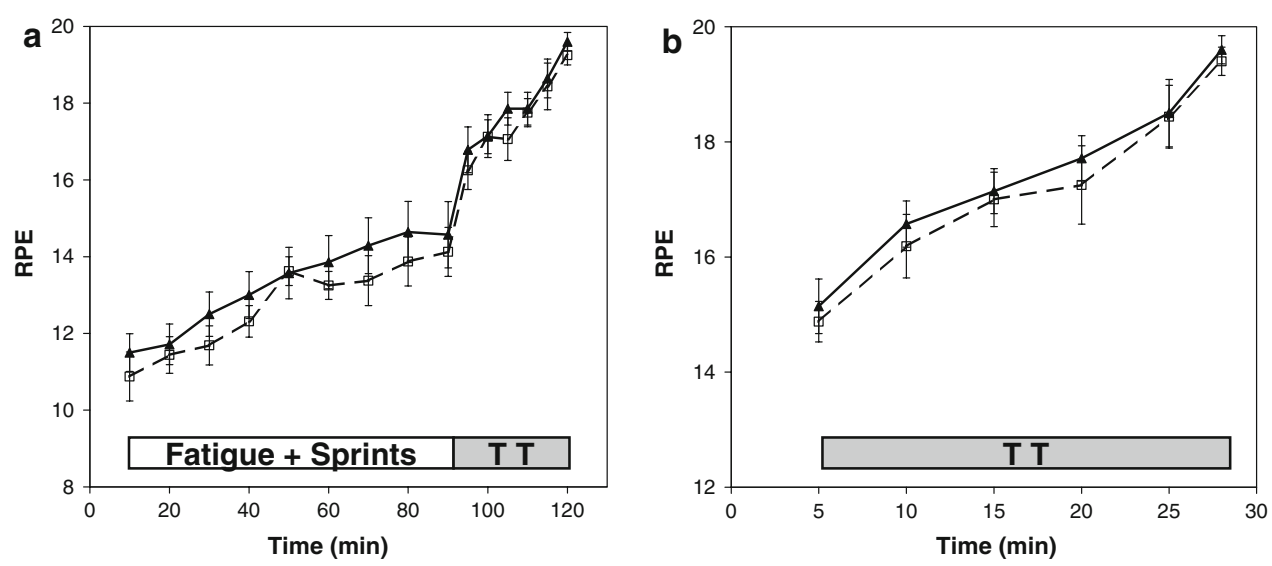

Fig. 7 Individual $(n=8)$ urinary $\mathrm{T} / \mathrm{epiT}$ ratios values during the PLA (dashed line, a) and TX (solid line, $\mathbf{b}$ ) trials

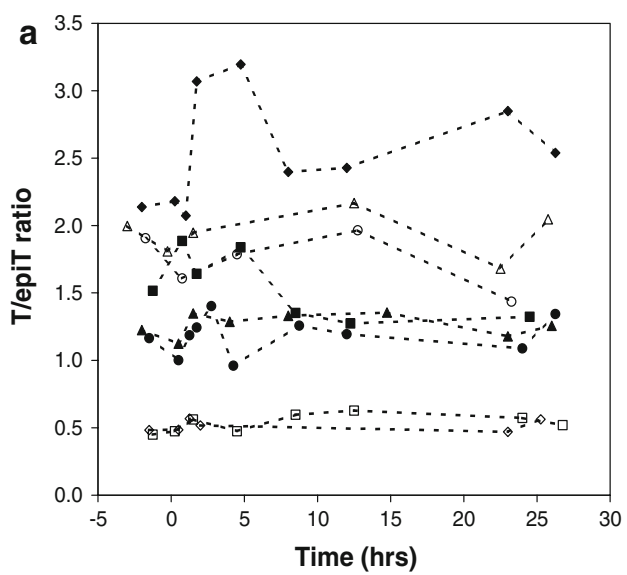

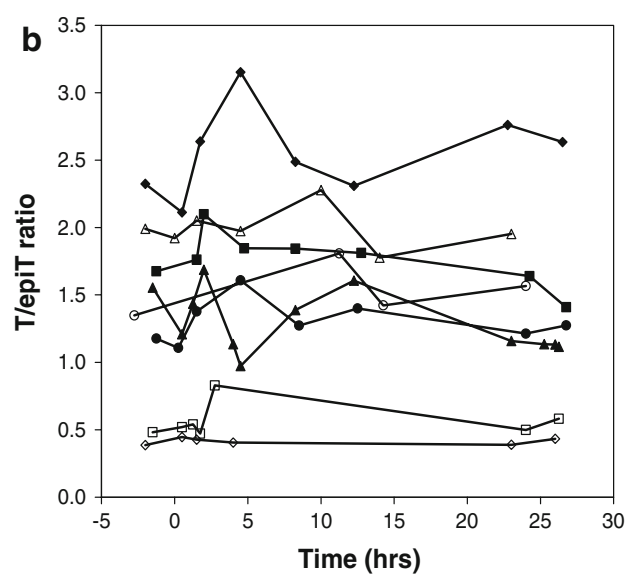

et al. 2000). As Synacthen ${ }^{\circledR}$ is a powerful enhancer of endogenous cortisol production (Wood et al. 1965), this drug is potentially very attractive to athletes seeking a potential increase in performance without taking any exogenous glucocorticoids.

\section{Appropriateness of the study protocol}

The protocol (Fig. 1) was completed as a double-blind crossover design and with within-subject controls. Each trial (TX and PLA), was separated by a period of 7-10 days since changes in training status are known to alter ACTH responsiveness (Luger et al. 1987) and also to allow a washout period of the medication. A familiarization trial was completed during the screening test session to enable the subjects to become familiar with the surroundings, to the performance of a $20-\mathrm{km}$ ergometer TT, to eliminate a "learning effect", and elevation of stress hormones due to unfamiliarity (Voigt et al. 1990). Four subjects had faster times to completion in the second trial, two did the $20-\mathrm{km}$ TT in the same times and the two others were slower. Thus, no "learning effect" was apparent in this study. The combination of a $90-\mathrm{min}$ fatiguing exercise followed by a $20-\mathrm{km}$ TT was selected as the aim of this study was to test how real athletes would use Synacthen ${ }^{\circledR}$ in competition. It is assumed that this substance would be administered on a short time period to boost the cortisol production right before an event. Thus, the potential positive effects of cortisol would allow a higher energetic state and better feelings for athletes during exercise. Administration of Synacthen ${ }^{\circledR}$ on a longer time period inducing a permanent high cortisol concentration in the body would be harmful to the athletes body composition as has been previously shown (Ferrando et al. 1999). Recently, in appropriate forums on internet, it appeared that athletes from different levels take up to $2.5 \mathrm{mg}$ of Synacthen ${ }^{\circledR}$. This massive dose has not been considered in our experiment, as we did not feel it appropriate in consideration of the minimal risk. However, the $0.25-\mathrm{mg}$ dose is the dose normally administered during studies of pituitary function and clearly elevated downstream hormones.

The exercise workload was controlled and normalized between subjects by setting the intensity of the fatiguing period and of the multiple sprint sessions at 50, 70 and $90 \%$ PPO, respectively. The superposition of the HR profiles of one subject during PLA and TX trials denotes that the participants exercised at the same relative intensity level in each trial (Fig. 2) allowing the comparison of the postfatigue performances (day 1) within subjects. The experimental conditions (laboratory temperature, timing of exer- 
cise and diet control) were monitored and were similar during both trials. Five sprints sessions were introduced in the fatigue phase to reproduce the stochastic nature of a cycling race (Schabort et al. 1998).

\section{Athletes population}

The selection of the studied population is a key criterion for a trial involving doping and performance assessment (Clarkson and Thompson 1997). Consequently, a $\mathrm{VO}_{2} \max$ greater than $57 \mathrm{ml} \mathrm{kg}^{-1} \mathrm{~min}^{-1}$ and a PPO higher than $360 \mathrm{~W}$, indicative of a high training level, were chosen as inclusion criteria. The eight volunteers involved in the trial had a mean $V \mathrm{O}_{2} \max$ of $63.7 \pm 4.5 \mathrm{ml} \mathrm{kg}^{-1} \mathrm{~min}^{-1}$ and a mean PPO of $428 \pm 54 \mathrm{~W}$ (Table 1) showing the suitability of the studied population compared to reference values of professional cyclists and sedentary people (Dunn et al. 1999; Mujika and Padilla 2001).

\section{Plasma ACTH and cortisol response}

The most important physiological effects of ACTH and Synacthen ${ }^{\circledR}$ involve the adrenal cortex and include the maintenance of adrenal weight and the control of adrenal corticosteroid synthesis and release. In healthy individuals, basal plasma cortisol fluctuates between 83 and $524 \mathrm{nmol}^{-1}$ in the morning and then decreases throughout the day (Weitzman et al. 1971). The mean basal cortisol concentrations measured on the eight volunteers (PLA, $478.6 \mathrm{nmol}^{-1}$ and TX, $517.0 \mathrm{nmol}^{-1}$ ) are in the top of the reference range. The response of the HPA axis to exercise has been extensively studied and it has already been demonstrated that basal pituitary-adrenal activity is elevated in endurance athletes (Duclos et al. 2003) due to an adaptive response of the HPA axis to repeated stimulations (Duclos et al. 2001). On day 1, the single IM injection of $0.25 \mathrm{mg}$ of Synacthen ${ }^{\circledR}$ induced a significant ACTH peak 30 min after injection in the TX group $\left(t_{2}\right.$, Fig. $\left.3 \mathrm{c}\right)$. Then, stimulation of the adrenal glands produced a prompt significant and standard increase in the cortisol profile (Fig. 3a) (Vogeser et al. 2001).

In the PLA group, the diurnal rhythm of cortisol can be observed with a slight decrease from $t_{0}$ till $t_{7}$. Subsequently, during the 20-km TT ( $t_{8}$ and $t_{9}$ ), and due to the physiological stress caused by the very intense effort, plasma cortisol increased. This increase allows a better availability of metabolic substrates to provide energy to the working muscles (Sapolsky et al. 2000). The metabolic stimulation of cortisol release in the PLA group is also illustrated by the ACTH peak measured during the 20-km TT $\left(t_{8}\right.$ and $t_{9}$, Fig. 3c). This phenomenon was not observed in the TX group as the peak cortisol concentration inhibits the corticotropin-releasing hormone (CRH) secretion by the hypo- thalamus through a negative feedback loop (Aguilera et al. 2007). The percentage of explained variance clearly showed that treatment (45\%) and sampling time (14\%) were the main parameters that influenced the cortisol variance. This result was expected as Synacthen ${ }^{\circledR}$ was used to stimulate cortisol production and exercise has been proven to modulate plasma cortisol concentration (Tremblay et al. 2005).

On day 2 of the trials, there was no difference in ACTH and cortisol profiles between the PLA and TX groups (Fig. 3c, d) indicating that the single IM injection received $24 \mathrm{~h}$ previously had no further influence on the HPA axis. The intense effort exhibited during the 20-km TT on day 2 stimulated the production of cortisol in both groups $\left(t_{3}\right.$ and $t_{4}$ ) without any significant difference between the groups.

Adrenal gland stimulation and plasma androgenic steroid hormones

The biosynthesis pathways of glucocorticoids and androgens are closely related with conversion of $17-\mathrm{OH}$ pregnenolone and 17-OH progesterone to DHEA and Adione, respectively. As shown previously (Horton and Tait 1966), Fig. $4 a, b$ illustrate an indirect but significant increase of DHEA and Adione plasma concentrations by stimulating the adrenal gland with Synacthen ${ }^{\circledR}$ (TX trial). Surprisingly, ANOVA of free plasma testosterone did show a significant difference between PLA and TX sessions. Indeed, adrenal steroids like DHEA and Adione are usually converted within the liver to $\mathrm{T}$ glucuronide and then excreted without leakage into the general circulation (Horton and Tait 1966). Thus, the variance between groups is mainly explained by subject variability $(59 \%)$ rather than treatment $(2 \%)$. The observed profiles of free plasma $\mathrm{T}$ diverge from previously published data showing a decrease in plasma $\mathrm{T}$, due to the antianabolic effect of raised cortisol on testicular steroidogenesis (Kicman et al. 1999; Schaison et al. 1978). This discrepancy could be a possible consequence of the different administered ACTH dose (1 mg on two consecutive days) used by Kicman et al. (1999). It should be noted that in PLA group, DHEA and Adione, which are anabolic hormones precursors, increased during high intensity effort $t_{2}{ }^{-}$ $t_{9}$ ). On the second day of the sessions and like cortisol, plasma DHEA, Adione and free $\mathrm{T}$ concentrations did not show any statistical difference between PLA and TX trials (data not shown).

\section{Cortisol and performance effect}

Cortisol is the final product of the HPA axis and a key hormone involved in multiple physiological pathways (Viru and Viru 2004). A high cortisol level is required in stressful situations to optimize muscular contraction by supplying an 
adequate quantity of energy substrates (Sapolsky et al. 2000) and to prevent inflammatory processes that could be deleterious to the organism's stress resistance. Thus, performing a 20-km TT while having plasma cortisol concentration at a very high level could be beneficial for athletes. As depicted in Fig. 3a, the protocol was correctly designed to perform the 20-km TT at the peak cortisol concentration during the TX trial. However, the time to completion of the 20-km TT did not show any statistical difference between the PLA and TX groups on either day of the trials (Fig. 5). These results suggest that a single IM injection of $0.25 \mathrm{mg}$ of Synacthen ${ }^{\circledR}$ is not sufficient to improve performance a few hours postinjection or the day after injection. Further, uncertainty regarding the ergogenic benefit stems from the fact that cortisol is a powerful proteolytic hormone primarily concerned with maintaining glucose concentration. The maintenance of blood glucose by cortisol often comes at the expense of body tissues since amino acids from protein breakdown can be diverted to the liver for gluconeogenesis or oxidation. In fact, prolonged elevated concentrations of cortisol are known to cause muscle wasting (Ferrando et al. 1999).

\section{Perceived exertion variation following Synacthen ${ }^{\circledR}$ IM injection}

To assess a psychological parameter of performance, the RPE scale was administered. This method provides information about "how" a person feels. As depicted in Fig. 6, the mean RPE values increased significantly with time for both the PLA and TX trials, on both days of the protocol. Comparison between PLA and TX values on day 1 did reveal a very small but significant difference with higher RPE values in the TX condition. No rational explanation has been found to justify this slight variation. Moreover, this observation does not support previous published results indicating a decreased feeling of fatigue with Synacthen ${ }^{\circledR}$ injection (Soetens et al. 1995). RPE values were also lower in the second trials (week 2) indicating a possible "learning effect" despite the familiarization trial. These results could be explained by one subject who did the TX trial on week 1 and who was extremely stressed with significantly higher HR and RPE values compared to the PLA session (data not shown). Without considering the data collected from this outlier, the endogenous cortisol stimulation following an acute IM injection of $0.25 \mathrm{mg}$ of Synacthen ${ }^{\circledR}$ does not seem to affect how the athletes feel during an intense physical effort $2 \mathrm{~h}$ after injection. At the end of the experiment, half of the subjects $(n=4)$ had a correct presumption when they were asked to guess in which session they were on treatment. This information was a good marker of how they felt and once again synthetic ACTH did not significantly influence their subjective feeling of fatigue.
Comparison with previously published data

Soetens et al. (1995) have previously examined performance after Synacthen ${ }^{\circledR}$ administration. However, some differences in the protocol have to be pointed out. Soetens et al. used steady-state exercise followed by a ramped test whereas our subjects were submitted to a fatiguing sprint session followed by a TT, which has been proven as a valid method for performance assessment (Palmer et al. 1996). Despite these differences in protocol, both studies showed no significant effect of Synacthen ${ }^{\circledR}$ on performance. The dose of synthetic ACTH in this study $(0.25 \mathrm{mg})$ was four times lower than the one used by Soetens et al. (1 mg) and the injection timing also differed as ours was given $30 \mathrm{~min}$ before exercise whereas theirs was given 45 min before. It is worth noting that the lower dose still produced a marked cortisol response but the intensity of this response could not be compared between studies as the hormone kinetics was not described by Soetens et al. Since the IM ACTH injection dose was higher in the former study, it is probable that subjects had higher cortisol concentrations during exercise, which could be an explanation for the difference observed regarding the feeling of fatigue between the two protocols.

Urinary glucuronides steroids as prospective secondary markers

Urine analyses revealed a significant raise in cortisol and metabolites glucuronides whereas androgens and related compounds did not fluctuate significantly between both sessions (data not shown). Kicman et al. (1999) showed that ACTH stimulation in eugonadal men induced a slight decrease in the urinary rate of $\mathrm{T}$ glucuronide but did not change the rate of EpiT glucuronide. These two steroids are of high interest as the ratio between $\mathrm{T}$ and EpiT (T/ epiT ratio) is a criterion in the determination of $\mathrm{T}$ abuse in sports (Palonek et al. 1995). About half of the EpiT is produced in the testis (Dehennin 1993) and little, if any, is derived from the peripheral metabolism of $\mathrm{T}$, Adione or DHEA (Brooks and Giuliani 1964). Our results showed that urinary EpiT glucuronide is not influenced by a single IM injection of synthetic ACTH and also that the T/epiT ratio remains constant (Fig. $7 \mathrm{a}$, b) within subject (CV $<30 \%$, WADA 2004) and independent of the experimental conditions. Moreover, it should be emphasized that the T/epiT ratio values of each subject are extremely comparable between the two sessions separated by 7-10 days. This observation has already been published (Catlin et al. 1997) and is important to consider in a longitudinal follow-up of athletes as proposed by sports organizations and antidoping laboratories. 


\section{Conclusions}

Although adrenocorticotropins and Synacthen ${ }^{\circledR}$ are banned by WADA, the efficacy of these substances on sporting performance is currently not well known. According to athletes anecdotal evidence, underground knowledge and products found in anti-doping agent's captures, Synacthen ${ }^{\circledR}$ seems to be a product that is currently used in cycling activity as an acute treatment. Regarding drug administration, it was important we used Synacthen ${ }^{\circledR}$ in the way an athlete would (right before exercise) and to determine whether its effects were significant a few hours after the injection (day $1 \mathrm{TT}$ ) and would last more than one day thus improving the subsequent performance (day 2 TT). As indicated by the results of this study, there is no obvious evidence of gains in physical performance and/or psychological well-being following an artificial boost of endogenous cortisol. It is well known that cortisol is involved in the stress response of an organism but, regarding performance enhancement, other factors that were not assessed might be necessary to optimize cortisol effects. Concerning possible detection in urine, none of the blood- and urinary-investigated parameters were conclusive to point out a manipulation of the endocrine system with synthetic ACTH.

It is important to note that it has not yet been determined whether multiple IM injections of synthetic ACTH could have a significant effect on performance and/or could produce a significant change in any blood or urine markers profiles. Therefore, although our present study and those of others would suggest that Synacthen ${ }^{\circledR}$ is not an effective doping agent, studies using more than one injection and/or higher dose need to be considered.

Acknowledgments We thank the subjects for their time and devotion to the study as well as Pro4bodyfuel for the supply of nutrition products. Additionally, we would like to acknowledge the Federal Office of Sports (BASPO) of Switzerland, Drug Free Sport New Zealand (DFSNZ) and the Swiss National Science Foundation (grant no. PBLA1-114241) for their financial support. The authors are grateful to Anna Rolleston, Cheryl Murphy, Carl Bradford and Darren Ellis for their technical and practical support and to the medical staff from the Adidas Sports Medicine Centre of Auckland (New Zealand) for their medical participation and assistance. The authors acknowledge also Dr Martial Saugy, director of the Swiss Laboratory for Doping Analyses, for his scientific advice and Dr Pierre-Edouard Sottas for his statistical analyses.

\section{References}

Abdi S, Datta S, Trescot AM, Schultz DM, Adlaka R, Atluri SL et al (2007) Epidural steroids in the management of chronic spinal pain: a systematic review. Pain Physician 10(1):185-212

Aguilera G, Kiss A, Liu Y, Kamitakahara A (2007) Negative regulation of corticotropin releasing factor expression and limitation of stress response. Stress 10(2):153-161. doi:10.1080/ 10253890701391192
Bogdanov AI, Yarushkina NI (2004) The role of adrenocorticotropic hormone in the inhibition of pain reactions in conscious rats. Neurosci Behav Physiol 34:575-578. doi:10.1023/B:NEAB. 0000028287.61380.05

Borg GA (1982) Psychophysical bases of perceived exertion. Med Sci Sports Exerc 14:377-381

Bourgogne E, Herrou V, Mathurin JC, Becchi M, de Ceaurriz J (2000) Detection of exogenous intake of natural corticosteroids by gas chromatography/combustion/isotope ratio mass spectrometry: application to misuse in sport. Rapid Commun Mass Spectrom 14(24):2343-2347. doi:10.1002/1097-0231(20001230)14:24< 2343::AID-RCM171>3.0.CO;2-Z

Brooks RV, Giuliani G (1964) Epitestosterone: isolation from human urine and experiments on possible precursors. Steroids 4:101116. doi:10.1016/0039-128X(64)90028-5

Burke LM, Hawley JA (2002) Effects of short-term fat adaptation on metabolism and performance of prolonged exercise. Med Sci Sports Exerc 34(9):1492-1498. doi:10.1097/00005768-20020 9000-00015

Catlin DH, Hatton CK, Starcevic SH (1997) Issues in detecting abuse of xenobiotic anabolic steroids and testosterone by analysis of athletes urine. Clin Chem 43:1280-1288

Clarkson PM, Thompson HS (1997) Drugs and sport: research findings and limitations. Sports Med 24(6):366-384. doi:10.2165/ 00007256-199724060-00003

Dehennin L (1993) Secretion by human testis of epitestosterone with its sulfoconjugate and precursor androgen 5-androstene-3b, 17adiol. J Steroid Biochem Mol Biol 44:171-177. doi:10.1016/09600760(93)90025-R

Duclos M, Corcuff JB, Pehourcq F, Tabarin A (2001) Decreased pituitary sensitivity to glucocorticoids in endurance-trained men. Eur J Endocrinol 144(4):363-368. doi:10.1530/eje.0.1440363

Duclos M, Gouarne C, Bonnemaison D (2003) Acute and chronic effects of exercise on tissue sensitivity to glucocorticoids. J Appl Physiol 94(3):869-875

Dunn AL, Marcus BH, Kampert JB, Garcia ME, Kohl HW 3rd, Blair SN (1999) Comparison of lifestyle and structured interventions to increase physical activity and cardiorespiratory fitness: a randomized trial. JAMA 281(4):327-334. doi:10.1001/jama.281.4.327

Dvorak J, Feddermann N, Grimm K (2006) Glucocorticosteroids in football: use and misuse. Br J Sports Med 40(Suppl 1):i48-i54. doi:10.1136/bjsm.2006.027599

Ferrando AA, Stuart CA, Sheffield-Moore M, Wolfe RR (1999) Inactivity amplifies the catabolic response of skeletal muscle to cortisol. J Clin Endocrinol Metab 84:3515-3521. doi:10.1210/ jc. 84.10 .3515

Fitch KD (1986) The use of anti-asthmatic drugs. Do they affect sports performance? Sports Med 3:136-150. doi:10.2165/00007256198603020-00004

Horton R, Tait JF (1966) Androstenedione production and interconversion rates measured in peripheral blood and studies on the possible site of its conversion to testosterone. J Clin Invest 45:301313. doi:10.1172/JCI105344

Johnson KL, Renn C (2006) The hypothalamic-pituitary-adrenal axis in critical illness. AACN Clin Issues 17:39-49. doi:10.1097/ 00044067-200601000-00006

Kicman AT, Coutts SB, Cowan DA, Handelsman DJ, Howe CJ, Burring $S$ et al (1999) Adrenal and gonadal contributions to urinary excretion and plasma concentration of epitestosterone in meneffect of adrenal stimulation and implications for detection of testosterone abuse. Clin Endocrinol (Oxf) 50:661-668. doi:10.1046/ j.1365-2265.1999.00742.x

Luger A, Deuster PA, Kyle SB, Gallucci WT, Montgomery LC, Gold PW et al (1987) Acute hypothalamic-pituitary-adrenal responses to the stress of treadmill exercise: physiologic adaptations to physical training. N Engl J Med 316:1309-1315 
Marfell-Jones M (2000) Kinanthropometric assessment. In: Bishop B, Hume P (eds) Guidelines for athlete assessment in New Zealand Sport, Sports Science New Zealand

Mazzarino M, Rossi F, Giacomelli L, Botrè F (2006) Effect of the systemic versus inhalatory administration of synthetic glucocorticoids on the urinary steroid profile as studied by gas chromatography-mass spectrometry. Anal Chim Acta 559:30-36. doi:10.1016/j.aca.2005.11.002

Medsafe, NZ (2006) Consumer Medicine Information

Mujika I, Padilla S (2001) Physiological and performance characteristics of male professional road cyclists. Sports Med 31(7):479487. doi:10.2165/00007256-200131070-00003

Nichols AW (2005) Complications associated with the use of corticosteroids in the treatment of athletic injuries. Clin J Sport Med 15:370-375. doi:10.1097/01.jsm.0000179233.17885.18

Nieman LK, Chanco Turner ML (2006) Addison's disease. Clin Dermatol 24(4):276-280. doi:10.1016/j.clindermatol.2006.04.006

Palmer GS, Dennis SC, Noakes TD, Hawley JA (1996) Assessment of the reproducibility of performance testing on an air-braked cycle ergometer. Int J Sports Med 17(4):293-298. doi:10.1055/s-2007972849

Palonek E, Gottlieb C, Garle M, Björkhem I, Carlström K (1995) Serum and urinary markers of exogenous testosterone administration. J Steroid Biochem Mol Biol 55:121-127. doi:10.1016/09600760(95)00146-Q

Phillipov G (1998) Effect of hypercortisolism and ACTH on the metabolism of cortisol. Exp Clin Endocrinol Diabetes 106:57-60

Polettini A, Marrubini Bouland G, Montagna M (1998) Development of a coupled-column liquid chromatographic-tandem mass spectrometric method for the direct determination of betamethasone in urine. J Chromatogr B Biomed Sci Appl 713(2):339-352. doi:10.1016/S0378-4347(98)00177-7

Reed MJ, Beranek PA, Cheng RW, Few JD, James VH (1987) The effect of cortisol or ACTH on plasma concentrations of free fatty acids. In Vivo 1:181-184

Ricketts ML, Verhaeg JM, Bujalska I, Howie AJ, Rainey WE, Stewart PM (1998) Immunohistochemical localization of type 111 betahydroxysteroid dehydrogenase in human tissues. J Clin Endocrinol Metab 83:1325-1335. doi:10.1210/jc.83.4.1325

Rook GA (1999) Glucocorticoids and immune function. Best Pract Res Clin Endocrinol Metab 13:567-581. doi:10.1053/beem.1999. 0044

Sapolsky RM, Romero LM, Munck AU (2000) How do glucocorticoids influence stress responses? Integrating permissive, suppressive, stimulatory, and preparative actions. Endocr Rev 21(1):5589. doi:10.1210/er.21.1.55

Schabort EJ, Hawley JA, Hopkins WG, Mujika I, Noakes TD (1998) A new reliable laboratory test of endurance performance for road cyclists. Med Sci Sports Exerc 30:1744-1750. doi:10.1097/ 00005768-199812000-00014
Schaison G, Durand F, Mowszowicz I (1978) Effect of glucocorticoids on plasma testosterone in men. Acta Endorcinol. 89:126-131

Sewer MB, Waterman MR (2003) ACTH modulation of transcription factors responsible for steroid hydroxylase gene expression in the adrenal cortex. Microsc Res Tech 61:300-307. doi:10.1002/ jemt. 10339

Soetens E, De Meirleir K, Hueting JE (1995) No influence of ACTH on maximal performance. Psychopharmacology (Berl) 118:260 266. doi:10.1007/BF02245953

Sottas PE, Saudan C, Schweizer C, Baume N, Mangin P, Saugy M (2008) From population- to subject-based limits of T/EPIT ratio to detect testosterone abuse in elite sports. Forensic Sci Int 174(23):166-172. doi:10.1016/j.forsciint.2007.04.001

Tamm J, Apostolakis M, Voigt KD (1966) The effects of ACTH and HCG on the urinary excretion of testosterone in male patients with various endocrine disorders. Acta Endocrinol (Copenh) 53:61-72

Thevis M, Bredehoft M, Geyer H, Kamber M, Delahaut P, Schanzer W (2006) Determination of Synacthen in human plasma using immunoaffinity purification and liquid chromatography/tandem mass spectrometry. Rapid Commun Mass Spectrom 20:35513556. doi: $10.1002 / \mathrm{rcm} .2774$

Tremblay MS, Copeland JL, Van Helder W (2005) Influence of exercise duration on post-exercise steroid hormone responses in trained males. Eur J Appl Physiol 94(5-6):505-513. doi:10.1007/ s00421-005-1380-x

Viru A, Viru M (2004) Cortisol—essential adaptation hormone in exercise. Int J Sports Med 25(6):461-464. doi:10.1055/s-2004821068

Vogeser M, Zachoval R, Jacob K (2001) Serum cortisol/cortisone ratio after Synacthen stimulation. Clin Biochem 34:421-425. doi:10.1016/S0009-9120(01)00251-X

Voigt K, Ziegler M, Grunert-Fuchs M, Bickel U, Fehm-Wolfsdorf G (1990) Hormonal responses to exhausting physical exercise: the role of predictability and controllability of the situation. Psychoneuroendocrinology 15:173-184. doi:10.1016/0306-4530(90) 90028-8

WADA (2004) WADA Technical Document: reporting and evaluation guidance for testosterone, epitestosterone, T/E ratio and other endogenous steroids, http://www.wada-ama.org.

WADA (2008) The 2008 prohibited list. The World Anti-Doping Code 2008. http://www.wada-ama.org

Weitzman ED, Fukushima C, Nogeire C (1971) Twenty-four-hour pattern of the episodic secretion of cortisol in normal subjects. J Clin Endocrinol Metab 33:14-22

Wilson H, Lipsett MB (1966) Metabolism of epitestosterone in man. J Clin Endocrinol 26:902-914

Wood JB, Frankland AW, James VH, Landon J (1965) A Rapid test of adrenocortical function. Lancet 191:243-245. doi:10.1016/ S0140-6736(65)91526-6 